\title{
Ein einfacher Immunfluoreszenztest zum Nachweis zytomegalievirusspezifischer (CMV-) IgM-Antikörper
}

\author{
H. W. Doerr \\ Instıtut fur Med. Virologie der Universität Heidelberg
}

\begin{abstract}
Zusammenfassung:
Mit der indirekten Immunfluoreszenz können in CMV-infizierten, lyopohilisierten Zellen, wie sie bei der Herstellung des KBR-Antigenes präpariert werden, die charakteristischen, intranukleärén Einschlußkörperchen nachgewiesen werden. Eine darauf basierende Testmodifikation zum Nachweis CMV-spezifischer IgM-Antikörper hat sich in der Erprobung an mehr als 100 Patienten, die mit Deckglaszellkulturen arbeitet, als gleichwertig mit der konventionellen Technik erwiesen.
\end{abstract}

\section{Schlüsse/wörter:}

Zytomegalie - Spez. IgM-Antikörper - Indirekte Immunfluoreszenz - Antigenpräparation.

\begin{abstract}
Summary:
Intranuclear inclusion bodies, which are typical of CMV infections, can be demonstrated by the indirect immunofluorescence technique using CMV infected cells, which have been lyophilized, as it is usual for the preparation of antigens for the CFT. A modified test system for the detection of CMV specific IgM antibodies working with this antigen has proven to be adequate to the conventional method, which uses slide tissue cultures.
\end{abstract}

Key words:

Cytomegalic inclusion disease - Specific IgM antibodies - Indirect immunofluorescence - Antigen preparation.

Neben der Komplementbindungsreaktion (KBR) stellt die indirekte Immunfluoreszenz (IFT) immer noch das am meisten angewandte sero-diagnostische Testverfahren zum Nachweis von CMV-IgM-Antikörpern dar. Gegenüber den neuerdings entwickelten Enzymimmunassays (ELISA) bietet sie den Vorteil, in das Antigen ,hineinsehen" zu können. Ihrer allgemeinen Anwendung steht die Erfordernis einer relativ aufwendigen Zell- und Virusanzüchtung für die Antigenpräparation unter standardisierten Bedingungen entgegen. Davon unabhängig ist eine Testmodifikation, die mit kommerziell erhältlichen, lyophilisierten Antigenchargen arbeitet und im folgenden vorgestellt wird:

\section{Material und Methoden}

Mit CMV lytisch infizierte und nach einer Glycinpufferinkubation lyophilisierte Humanfibroblasten*) werden pro Charge in $1 \mathrm{ml} \mathrm{A}$. bidest. resuspendiert, mit Veronalpuffer (VP; pH 7,2) 1:6 verdünnt und $100 \mu \mathrm{l}$ der Suspension auf Spezial(kunststoff)deckgläschen ${ }^{* *}$ ) aufgetropft. Nach Antrocknung in einem Umluftbrutschrank bei $+37^{\circ} \mathrm{C}$ erfolgt eine Fixation mit $1 \%$ Formalin (in VP) für 5 Min. Die Deckgläschen werden danach

-) Behringwerke, Marburg (CMV-Antigen für die KBR)

-) Fa. Medac, Hamburg 
gründlich in VP gewaschen und sind dann für den Test gebrauchsfertig:

Pro Gläschen werden je $100 \mu$ l der Patientenseren (1:20 und $1: 40$ in VP verdünnt) gleichmäßig aufgetropft und bei $+37^{\circ} \mathrm{C}$ in der feuchten Kammer für $3 \mathrm{~h}$ inkubiert. Anschließend erfolgt für $20 \mathrm{Min}$. ein Waschvorgang bei langsamer ständiger Bewegung der Waschlösung (VP + 0,1\% Tween 20) über den .Präparaten (automat. Schaukelplatte). Zum Nachweis des SerumIgM, das mit dem Träger-fixierten Antigen reagiert hat, verwendet man pro Deckgläschen je $50 \mu l \mu$-kettenspezifische, fluoreszeinkonjugierte Anti-IgM-Antikörper***) (1:40 in VP verdünnt) bei einer Inkubation von 30 Min. bei Raumtemperatur. Nach einem erneuten gründlichen Waschvorgang ( $\geq 2 \mathrm{~h}$ ) sind die Präparate fertig und können unter einem Fluoreszenzmikroskop ausgewertet werden.

\section{Ergebnisse und Diskussion}

Abb. 1 zeigt die typische Fluoreszenz der Kerneinschlüsse von CMV-infizierten Humanfibroblasten, die, wie oben angegeben, präpariert und mit einem $\mathrm{CMV}$ IgM-positiven Serum beschichtet worden sind. Sie läßt sich einwandfrei von der unspezifischen Zytoplasmafluoreszenz unterscheiden.

Die neue Testmodifikation wurde in die laufende Labordiagnostik unseres Institutes eingesetzt. Die Serumproben von über 100 Patienten, darunter $15 \mathrm{CMV}$-IgMpositive Fälle, zeigten in den getesteten Verdünnungsstufen eine völlige Übereinstimmung der Ergebnisse zur konventionellen Arbeitstechnik (Anzüchtung CMV-infizierter Zellen auf Deckgläschen) (4). Wegen begrenzter Haltbarkeit sollte das einmal resuspendierte Antigen unmittelbar in die Tests eingesetzt werden.

Bei jedem positiven Resultat ist die Bildung von IgMIgG-Immunkomplexen im Patientenserum auszuschließen, die wegen der hohen Populationsdurchseuchung mit CMV in dieser Testanordnung eine falsch positive CMV-IgM-Spezifität vortäuschen können. Wir überprüfen dies mit einem Rheumafaktor-Latex-Agglutinationstest. Bei positivem Ausfall muß der Rheumafaktor, besser noch die lgG-Fraktion aus dem Testserum absorbiert werden (3). Ein falsch positives Ergebnis des CMV-IgM-Tests durch antinukleäre Antikörper läßt sich oft an einem unterschiedlichen Fluoreszenzmuster erkennen; gegebenenfalls kann man auch ein Kontrollantigen (nicht oder mit einem anderen Virus infizierte Zellen) einsetzen.

Da CMV-IgM-Antikörper auch unspezifisch im Verlaufe anderer Virusinfektionen reaktiviert werdèn können

-.) Fa. Dako, Kopenhagen

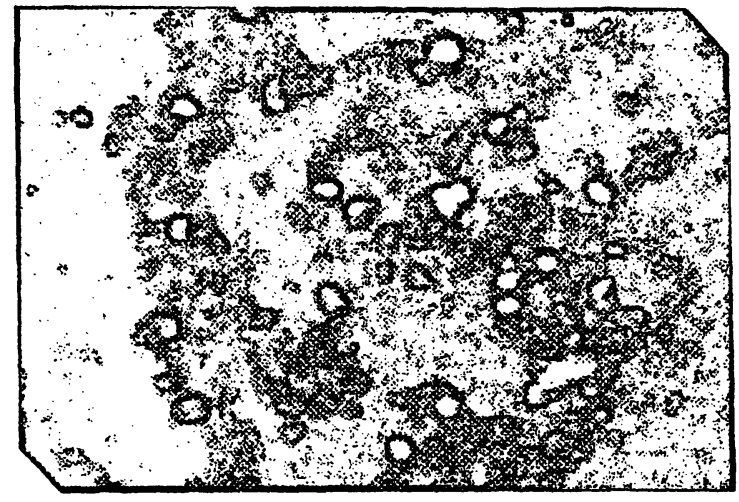

Abb. 1:

Kern(einschlußkörperchen)fluoreszenz und unspezifische Zytoplasmafluoreszenz von CMV-infizierten Zellen nach Lyophilisation und Resuspendierung. Die Zelltrümmer wurden bei $+37^{\circ} \mathrm{C}$ angetrocknet und mit $1 \%$ igem Formalin fixiert

(2) bzw. Kreuzreaktionen bei Epstein-Barr-Virusinfektion auftreten (4), sollte ihre Bestimmung stets ergänzend zu einer bewährten Standardmethode (z. B. KBR) erfolgen, um eine adäquate Befundinterpretation sicherzustellen (1).

Schrifttum:

1. DOERR, H.W., DARAI, G.: Fluoreszenzserologischer Nachweis von Virusinfektionen. Lab.med. 2, 209-214 (1978).

2. DOERR, H.W., GEISEN, H. P., KAPP, M., SCHMIDT, W.: Antikörperentwicklung nach Rötelnvakzination im Wochenbett bei primär Röteln-seropositiven Frauen. Nachweis voǹ Antigen-spezifisch (Röteln) und nicht spezifisch stimulierten AntikörNachweis von Antigen-spezifisch (Roum) Disch. Med. Wschr., 105, 1316-1320 (1980).

(1980). 3. GEISEN, H. P., FRANK, R., DOERR, H. W., ENDERS, G.: Simple method to detect
virus-specific IgM antibodies in patient's serum samples after immunosorption of virus-specific IgM antibodies in patient's serum samples after immunosorption
immunoglobulins $G$ and $A$. Med. Microbiol. Immunol. 167, 77-82 (1979).

4. SCHMITZ, H., HAAS, R.: Determination of different cytomegalovirus immunoglobulins ( $\lg G, \lg M, \lg A)$ by immunofluorescence. Arch. ges. Virusiorsch. $37,131-140$ (1972).

Anschrift des Verfassers:

Priv.-Doz. Dr. med. H. W. Doerr

Facharzt für Laboratoriumsmedizin

Institut für Med. Virologie der Universität

Im Neuenheimer Feld 324

D-6900 Heidelberg 